\title{
Acute Cholecystitis: Diagnostic Pitfall and Timing of Treatment
}

\author{
Pasquale Cianci, Nicola Tartaglia, Alberto Fersini, \\ Sabino Capuzzolo, Libero Luca Giambavicchio, \\ Antonio Ambrosi and Vincenzo Neri
}

Additional information is available at the end of the chapter

http://dx.doi.org/10.5772/67549

\begin{abstract}
Objective: Cholelithiasis represents a very frequent health problem with higher prevalence in developed countries. The aim of this chapter is to underline, also by submitting our surgical experience, some diagnostic deceptions and the timing of treatment.
\end{abstract}

Methods: The presentation of 42 patients admitted in our institution (September 2012/ September 2014) with the diagnosis of acute pancreatitis allows to identify two different clinical forms of acute biliary pancreatitis: the pancreatic pattern and biliary pattern. Moreover, the evaluation of another 42 patients observed in our institution (September 2014/September 2016) with acute cholecystitis should show our treatment program. Also, we added the analysis of our previous research, regarding acute cholecystitis, already published: difficult cholecystectomy, antegrade dissection in laparoscopic cholecystectomy, postoperative morbidity, laparoscopic approach in cirrhotics, finally the robotic experience.

Results: Clinical features, laboratory, and imaging exams should identify, into acute biliary pancreatitis, two clinical forms as biliary pattern and pancreatic pattern for different therapeutic approach. The treatment chosen for acute cholecystitis is early laparoscopic cholecystectomy within 24-72 hours. Severe, complicated acute cholecystitis can require urgent surgical intervention.

Conclusion: Acute cholecystitis encompasses clinical forms with various degree of severity and several clinical courses. The treatment is focused on early cholecystectomy with various and different management strategies, suitable to the specific pathological conditions.

Keywords: acute cholecystitis, acute pancreatitis, cholelithiasis, cholecystectomy, laparoscopic approach 


\section{Introduction}

Cholelithiasis represents a very frequent health problem. The prevalence of cholelithiasis is higher in the developed regions such as Europe and North America in comparison to the developing regions of the world (Africa, Middle East, China, India, Far East). On average, gallstone disease affects $10-15 \%$ of the adulthood population in the age of majority $[1,2]$. The cost of gallstone disease has high social, administrative, and economic impact as interference with work activities, home care, hospital admission, and so on. Mortality rate for gallstone disease reaches $0.6 \%$, thanks to the reduction of more than $50 \%$ over the last 60 years [3]. Gallstone disease in its evolution involves acute cholecystitis and some risks of complications such as gallstone-related pancreatitis and cancer [4, 5]. Moreover, cholecystectomy morbidity encompasses various and diversified pathological conditions especially in severe inflammatory circumstances. We underline the problems connected with insufficient preoperative evaluation. Complications can be divided in intraoperative and postoperative. Intraoperative morbidity includes bile duct injury, gallbladder perforation, bleeding, and bowel perforation. Postoperative complications consist of infection and dehiscence of surgical incision, subhepatic abscess, residual choledocolithiasis, postcholecistectomy syndrome, umbilical hernia. Currently, cholecystectomy morbidity rate reaches $8.7-9.5 \%$ with up $15 \%$ in so-called difficult cholecystectomy. Among these complications is in evidence the bile duct injury which causes great impact on patient outcomes and requires usually complex and various procedures of repair: endoscopy, surgery, operative biliary radiology [6].

\section{Etiology and pathogenesis of gallstones}

Various etiological conditions and risk factors can cause gallstone disease. We can underline the gender, age, obesity, fast weight loss, alcohol use, diabetes, pregnancy, hypertriglyceridemia, and so on. The study of pathogenesis of gallstones can identify all etiological factors. The majority of gallstones are non-pigmented stones which are composed of cholesterol $(75 \%$ of cases). The cholesterol is retained in solution by an unsteady balance among levels of phospholipids, bile acids, and cholesterol (Admirand's triangle) [7]. This balance can be disrupted by several factors: cholesterol supersaturation in bile, crystal nucleating factors because cholesterol supersaturates tends to precipitate and crystallize, impairment of gallbladder functions as motility, absorbtion, secretion, finally impaired enterohepatic circulation of bile acids that changes the balance of Admirand's triangle. In summary, cholesterol stones are caused by cholesterol iperproduction, large cholesterol-phospholipid vesicles, crystal precipitation (cholesterol monohydrate crystal) [8]; moreover, by calcium nucleation, and other nucleating factors as mucin glycoproteins, immunoglobulins, and so on. In addition, impairment of gallbladder functions plays a significant role: decrease of motility with stasis as in prolonged fasting and parenteral nutrition, diabetic disease, long-term somatostatin therapy, alteration of absorptive, secretive activity with increase of water reabsorption. Finally, the reduction of intestinal reabsorption of bile acids in the entero-hepatic circulation is also in evidence. Crystallizations of cholesterol within bile form biliary sludge and biliary sludge can be considered a common precursor of the gallstones. Pigmented stones consist of calcium-bilirubinate. These stones 
are due to solubilization of unconjugated bilirubin with precipitation. There are two types: black and brown. Black stones reach about $15-20 \%$ of global biliary stones. They are caused and occur in several diseases: hemolytic disorders (increased red blood cell destruction), liver diseases, cirrhosis (abnormal metabolism of hemoglobin), distal ileal resection (reabsorption of bile salts), and long-term total parenteral nutrition (TPN). Commonly these stones form in gallbladder. Brown stones, on the contrary, are found in biliary ducts as primary common bile duct (CBD) stones. These stones are associated with infection in bile ducts. Bacteria (Escherichia coli, Klebsiella frequently) produce bacterial beta-glucuronidase; consequently deconjugated bilirubin, not hydrosoluble, forms calcium bilirubinate. Biliary infections are commonly associated with biliary ducts stenosis, ampullary stenosis, abnormal sphincter of Oddi, sclerosing cholangitis, cirrhosis.

\section{Epidemiology and pathophysiology of acute cholecystitis}

Symptoms or complications of gallstones can develop in 1-2\% of the patients for years [9]. Clinical presentation of cholelithiasis can be various: in the majority of cases (60-80\%) lithiasis stay on long asymptomatic or for the patient's whole life and its detection could be incidental. Symptoms of different degree, mild or severe for advanced complications occur in the $20-40 \%$ of patients. Acute cholecystitis is the very frequent surgical entity that occurs in 15-20\% of patients with symptomatic disease. Cholecystitis could be caused by obstruction of the cystic duct by a gallstone with the same pathogenesis of biliary colic. The obstacle of bile outflow from gallbladder causes its wall distention and wall inflammation. This pathological condition may develop in different ways. In the severe cases (10-18\%), the prolonged and complete obstruction causes extension of parietal flogosis resulting in disturbance of blood perfusion and necrosis. In the favorable cases, which are the majority, the stone moves, obstruction resolves, and inflammation may regress. In the acute cholecystitis, bacterial superinfection can occur in 50\% of cases with positive bile culture (Escherichia coli, Klebsiella, Enterobacter, etc.) [10]. We can believe that acute cholecystitis starts as inflammatory disease without bacterial infection. Recently more complex pathogenesis has been hypothesized in acute cholecystitis. Acute cholecystitis should be produced with the addition of irritating factors of gallbladder mucosa to the blockage of the cystic duct. Lysolecithin has been used in experimental setting as irritant; but lysolecithin comes by catalyzation from lecithin, normal constituent of bile, by phospholipase A. Trauma of impacted gallstone may cause the release of this enzyme [11]. Moreover, lysolecithin was found in the gallbladder with acute inflammation [12]. Gallbladder flogosis should be worsened by further inflammatory mediators such as prostaglandins, which play an important role in functional activity of gallbladder (motility, fluid absorption, etc.) [13]. In summary, prolonged obstruction of gallbladder neck leads the increase of intraluminal pressure, with venous congestion, impaired blood supply, and lymphatic drainage. Damage of gallbladder wall (edema, intramural haemorrhage) and secondary bacterial infection complete the pathological features. Acalculous cholecystitis is acute inflammatory disease associated with right upper abdominal quadrant pain, leucocitosis, thickened wall without gallstones (ultrasonography (US) findings). Most frequently, it happens in patients with severe disease such as severe burns, trauma, major surgery, long-term TPN; frequently cholecys 'titis can develop with high morbidity and mortality [14]. 
In the acute acalculous, cholecystitis probably can play a role of the bile stasis (fasting, narcosis) causing distension of wall, impaired blood supply, necrosis. Increased viscosity by dehydration and intestinal dynamic occlusion produces sludge formation and bacterial overgrowth in the gallbladder. US shows gallbladder wall thickening, sludge, pericholecystic fluid.

\section{Clinical presentations of gallstone disease}

Gallstones disease can present and develop in the wide clinical range. Asymptomatic disease can be detected incidentally. The absence of symptoms is linked to the mobility of stones that will not obstruct the cystic duct. The presence of gallstones, although asymptomatic for a prolonged time, can develop in symptomatic disease with various clinical entities. Most simple and frequent presentation is biliary colic characterized by abdominal pain localized in right upper abdominal quadrant, nausea, vomiting, frequently irradiating to the right shoulder. Usually the colic lasts a few hours. Asymptomatic patients can develop symptomatic disease in $20-30 \%$ of cases in the long term (20 years). The clinical developments of gallstone disease encompass several presentations: biliary colic, acute cholecystitis (with various degree of severity such as gangrene, emphysematous cholecystitis, perforation, cholecystoenteric fistula, gallstone ileus), choledocolithiasis, cholangitis, biliary pancreatitis, gallbladder carcinoma. The significant clinical problem is the surgical indication of cholecystectomy for patients with asymptomatic gallstone. The overall likelihood of clinical appearance for asymptomatic patients should be about $30 \%$ but we have to insert it and evaluate it in specific conditions: demographic, pathophysiological, and clinical. Another relevant information for the surgical indication choice is the incidence of postoperative morbidity of cholecystectomy. From the literature, overall morbidity (minor and major) of cholecystectomy in the laparoscopic era for uncomplicated gallstone disease in patients without comorbidity is very low: overall complication rate is $1.5 \%$ and the mortality rate is less than $0.1 \%$ [15]. In summary, surgical treatment is the first choice in the patients with symptoms, cholecystitis, and gallbladder stone-related complications. Moreover, nowadays, the surgical indication for patients completely asymptomatic is debatable and not well defined. We can identify several clinicalpathological conditions without clear and evident clinical appearance in which laparoscopic cholecystectomy should be indicated: patients with mild clinical appearance such as intense discomfort in the right upper quadrant, nausea vomiting, biliary colic because considerable risk for developing complications, young patients because high likelihood to develop in later years symptoms or/and complications, patients with pigmented stones caused by hemolytic disorders (increased red blood cells destruction) because the risks linked to this pathology in case of gallstone-related complications, patients with clearly established gallbladder dysfunction that frequently develops symptomatic disease (25-30\% of cases) [16], patient with large stones $(>2 \mathrm{~cm}$ ) for high risk to develop cholecystitis, patients with porcelain gallbladder (calcifications in the wall) because of the risk of gallbladder cancer (5-10\%).

\subsection{Common clinical presentation of acute cholecystitis}

Patients with mild symptomatic gallstone disease such as recurrent biliary colic or mild postprandial discomfort can develop in about $20 \%$ of cases acute cholecystitis. This path- 
ological evolution commonly is connected with obstruction by stones of gallbladder neck or cystic duct. The time duration of the obstructive condition (short or long time) can lead to decrease and resolution of inflammatory process or, on the contrary, to wall distension, impaired blood supply, ischemia, necrosis of gallbladder (severe cases 15-20\%). In about $50 \%$ of cases, commonly in the prolonged impairment of bile outflow from the gallbladder, bacterial infection adds to flogistic process. In acute cholecystitis, patients complain of severe pain in abdominal right upper quadrant and overall the clinical presentation is overlappable to biliary colic but the characteristic pain is on the contrary prolonged greater than 4-6 hours. Usually fever, nausea, anorexia, and vomiting join and the pain should be referred to right shoulder or back. Frequently the patients refer previous episodes of biliary colic, or fatty food ingestion few hours before the onset of colic. Clinical observation shows the suffering patient with fever, tachycardia, nausea, emesis, anorexia, and inhibition or diminution of the respiratory movement of right upper quadrant and epigastric area of abdominal wall. On physical examination can be seen right upper quadrant tenderness of varying degree and positive Murphy's sign with increased discomfort and/or inspiratory arrest while, palpating right upper quadrant, the patient is invited to make deep inspiration. A positive sign shows sensitivity of $97 \%$ and specificity of $93 \%$ [17]. In some cases (about $30 \%$ ), acute cholecystitis can develop discrete extension of inflammation outside gallbladder wall causing local peritonitis with involvement of omentum and adjacent organs that forms a flogistic mass, palpable in upper right quadrant. Leukocytosis is an almost constant laboratory finding characterized by the white cell count increase, connected with the severity of the disease.

\subsection{Deceptions of clinical diagnosis of acute cholecystitis}

Cholelithiasis is the most frequent cause of acute pancreatitis. Clinical diagnostic difficulties may arise in the context of acute biliary pancreatitis. The major clinical problem is to distinguish clinical forms of hyperamylasemia, associated with severe abdominal pain, physical signs of upper abdominal tenderness and guarding based on acute biliary tract disease (acute cholecystitis, cholangitis, etc.) from acute biliary pancreatitis with evident pancreatic involvement. In the context of acute biliary pancreatitis may merge acute abdominal diseases different to each other; but they have in common hyperamylasemia and acute/severe upper abdominal pain and abdominal wall guarding. The correct diagnosis and distinction between moderate or severe acute biliary pancreatitis with hyperamylasemia, evident pancreatic involvement, severe upper abdominal pain/abdominal wall guarding, and acute biliary tract disease (cholecystitis, cholangitis, etc.) with hyperamylasemia, severe upper abdominal pain, abdominal wall guarding, minimal, or mild pancreatitis, allows to follow different therapeutic program overall in regard to timing of surgery [18]. Our aim is to define clinical and laboratory differentiation between these two clinical manifestation regarding the choice of therapeutic program. The presentation of consistent and appropriate experience should clarify some diagnostic difficulties, within the acute biliary pancreatitis, between two clinicalpathological forms different but confusable. We have evaluated 42 patients admitted in our Institution in the period September 2012/September 2014. The admission diagnosis was acute pancreatitis, based on first basic clinical and laboratory evaluation. Demographic features: male 26, female 16, mean age 64 years (range: 89-27 years). Signs and symptoms of 42 patients at the admission are reported in (Figure 1). 


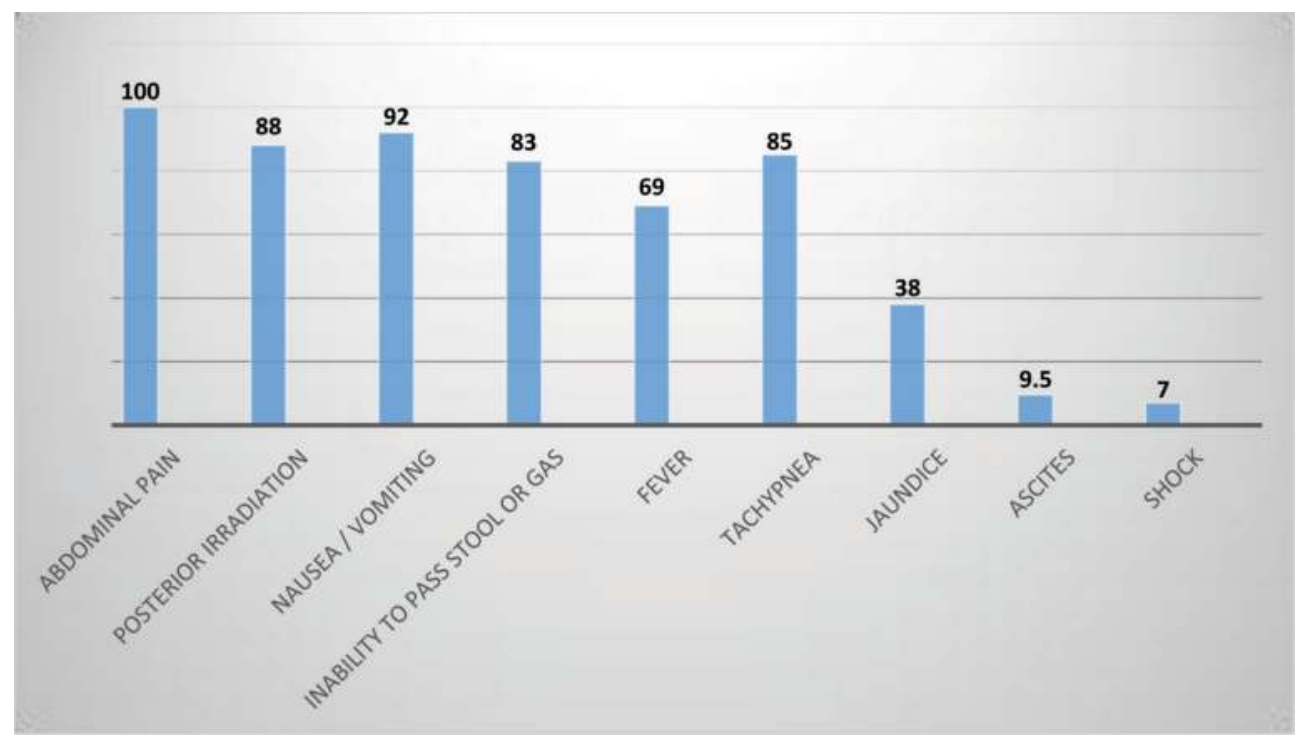

Figure 1. Frequency (\%) of signs and symptoms in patients with diagnosis of acute pancreatitis.

In the first phase of the study, the patients have been divided by etiology of acute pancreatitis. The majority of cases (30 pts. 71\% - Group A) shows biliary etiology, based on the detection with imaging study (US) of biliary lithiasis. In seven patients (Group B-16.6\%), the clinicalanamnestic criteria show the alcoholic etiology (prolonged alcohol abuse). The other five patients (Group C $-11.9 \%$ ) have been classified as acute pancreatitis patients with unknown etiology. The patients subdivided following the etiological criteria (Groups A, B, C) have been evaluated regarding to severity of disease with Ranson criteria, pancreatic involvement with CT severity index (Balthazar), and finally likelihood of biliary etiology with Blamey criteria using clinical and laboratory data (age, sex, amylase, alkaline phosphatase, ALT) [19-22]. The important section of this study concerns the biliary pancreatitis. Within 30 patients with initial diagnosis of acute biliary pancreatitis (Group A), we have identified two subgroup: the first subgroup A1 that encompasses 18 patients with acute biliary pancreatitis with moderate/ severe pancreatic involvement and the subgroup A2 that includes 12 patients with acute biliary disease and minimal pancreatic involvement based on transient hyperamylasemia. The aim of this subdivision and comparison is to identify, by laboratory and imaging study, two different clinical forms of acute pancreatitis: the pancreatic pattern (A1) and the biliary pattern (A2). The patients have been subdivided in three groups following the etiology criteria: biliary (Group A), alcoholic (Group B), and undefined pancreatitis (Group C). First, we can evaluate if there are differences among the groups of patients regarding clinical severity (Ranson score), degree of pancreatic involvement (CT severity index-Balthazar), and finally the likelihood of biliary etiology (Blamey score). The evaluation of clinical severity (Ranson score) between the group A (biliary) and group B (alcoholic) shows no differences with Student's t-test: $t=0.1375$ $<t_{0.05}=1.6896$. Because of the low number of cases in our groups, we have also employed the Kolmogorov-Smirnov test for the comparison of clinical severity (Table 1). 


\begin{tabular}{ll}
\hline Group A ${ }^{\mathrm{a}}$ versus Group B & $\mathrm{D}=0.205<\mathrm{D}_{0.05}=0.554$ \\
Group A versus Group C & $\mathrm{D}=0.634<\mathrm{D}_{0.05}=0.640$ \\
Group B versus Group C & $\mathrm{D}=0.429<\mathrm{D}_{0.05}=0.800$
\end{tabular}

*Kolmogorov-Smirnov test.

aGroup A: biliary.

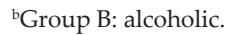

cGroup C: undefined.

Table 1. Comparison of clinical severity between group A, group B, and group C*.

The results of the severity disease comparison (CT severity index) show that there are not statistically significant differences between group A versus group B and between group B versus group $\mathrm{C}$. The comparison between group $\mathrm{A}$ versus group $\mathrm{C}$ shows also no differences (empirical $\mathrm{p}$ value $<$ theoretical $\mathrm{p}$ value) even if in the group $\mathrm{C}$ there are mild pancreatitis and in group A there are severe pancreatitis. The comparison among the three groups of degree of pancreatic and extrapancreatic damage (CT severity index-Balthazar) demonstrates that, even in this area, there are not statistically significant differences (Table 2).

Finally the evaluation of the predictive accuracy of biliary etiology based on clinical data (Blamey score) among the three groups did not provide effective results for the early definition of the biliary etiology because of no statistical differences (Table 3).

\begin{tabular}{ll}
\hline Group A versus Group B $^{\mathrm{b}}$ & $\mathrm{t}=0.4345<\mathrm{t}_{0.05}=1.609$ \\
Group A versus Group C & $\mathrm{t}=0.2884<\mathrm{t}_{0.05}=1.6939$ \\
Group B versus Group C & $\mathrm{t}=0.2006<\mathrm{t}_{0.05}=1.8595$
\end{tabular}

*Student's t-test.

${ }^{a}$ Group A: biliary.

bGroup B: alcoholic.

'Group C: undefined.

Table 2. Comparison of CT severity index between group A, group B, and group $\mathrm{C}^{*}$.

\begin{tabular}{ll}
\hline Group A $A^{\mathrm{a}}$ versus Group B & $\mathrm{t}=0.0568<\mathrm{t}_{0.05}=1.6896$ \\
Group A versus Group C & $\mathrm{t}=0.9195<\mathrm{t}_{0.05}=1.6924$ \\
Group B versus Group C & $\mathrm{t}=0.8594<\mathrm{t}_{0.05}=1.8125$ \\
\hline${ }^{\text {*Student's t-test. }}$ & \\
${ }^{\mathrm{a}}$ Group A: biliary. & \\
${ }^{\mathrm{b}}$ Group B: alcoholic. & \\
cGroup C: undefined. & \\
\hline
\end{tabular}

Table 3. Comparison of Blamey score between group A, group B, and group C*. 
To identify the two subgroups A1 (pancreatic pattern) and A2 (biliary pattern) within the group A (acute biliary pancreatitis) have been used clinical features, laboratory, instrumental tests (imaging), therapeutical procedures employed. We have verified if there are statistically significant differences between group A1 and group A2 with respect to leukocytosis, amylasemia, bilirubin, aspartate aminotransferase (AST), alanine aminotransferase (ALT), alkaline phosphatase, clinical severity score (Ranson), modified CT severity index (Balthazar). The purpose of the study is to identify clinical or instrumental criteria for detection of pancreatic pattern versus biliary pattern in acute biliary pancreatitis. In the statistical evaluation, leukocytosis, amylasemia, and alkaline phosphatase did not show differences between the two groups. On the contrary, there are differences for bilirubin, AST, and ALT. The results of comparison, among subgroups A1 and A2, of clinical severity score (Ranson) and modified CT severity index (Balthazar) are different with statistical significance with Student's t-test but not following Kolmogorov-Smirnov test (Table 4).

Finally, we have compared the results of clinical severity score and modified CT severity index respectively within the subgroup A1 (Figure 2) and subgroup A2 (Figure 3).

In the subgroup A1 (pancreatic pattern), the results of two scores are overlappable (covariance $=0.000177>0$ ). Otherwise in the subgroup A2, the data of the two scores are discordant. The results of this section of our study allow several considerations. First, we can identify in the group A (acute biliary pancreatitis) two subgroups: A1 (pancreatic pattern) and A2 (biliary pattern). In the subgroup A2, the pancreatic involvement (valued with modified CT severity index) was mild (pancreatic edema); on the contrary, in the subgroup A1, the pancreatic damage was moderate/severe or severe (Grade C2, D3); the difference between the two groups is statistically significant with Student's t-test not with Kolmogorof-Smirnov test. The clinical severity (Ranson score) was comparable in both groups and of middle level. The comparison of bilirubin, AST, ALT shows impairment significant in the subgroup A2; not significant the differences for amylasemia, leukocytosis, and alkaline phosphatase. The therapeutic program followed the indication of clinical evaluation. The first approach is based on medical treatment: fluid-electrolyte replacement, control of pain, nutrition, control of papillary flow and, if necessary removal of persistent papillary obstacle. Patients (18) with

\begin{tabular}{lll}
\hline & Student's t-test & Kolmogorov-Smirnov test \\
\hline White blood cells & $\mathrm{t}=0.2918<\mathrm{t}_{0.05}=1.7011$ & $\mathrm{D}=0.22<\mathrm{D}_{0.05}=0.500$ \\
Amylasemia & $\mathrm{t}=0.8754<\mathrm{t}_{0.05}=1.7011$ & $\mathrm{D}=0.203<\mathrm{D}_{0.05}=0.500$ \\
Bilirubin & $\mathrm{t}=2.0192<\mathrm{t}_{0.05}=1.7011$ & $\mathrm{D}=0.72<\mathrm{D}_{0.05}=0.500$ \\
AST & $\mathrm{t}=2.1664<\mathrm{t}_{0.05}=1.7011$ & $\mathrm{D}=0.67<\mathrm{D}_{0.05}=0.500$ \\
ALT & $\mathrm{t}=8.7062<\mathrm{t}_{0.05}=1.7011$ & $\mathrm{D}=0.78<\mathrm{D}_{0.05}=0.500$ \\
Phosphatase & $\mathrm{t}=0.6253<\mathrm{t}_{0.05}=1.7011$ & $\mathrm{D}=0.39<\mathrm{D}_{0.05}=0.500$ \\
Ranson's score & $\mathrm{t}=1.8477<\mathrm{t}_{0.05}=1.7011$ & $\mathrm{D}=0.363<\mathrm{D}_{0.05}=0.500$ \\
Balthazar's index & $\mathrm{t}=1.8585<\mathrm{t}_{0.05}=1.7011$ & $\mathrm{D}=0.416<\mathrm{D}_{0.05}=0.500$ \\
\hline
\end{tabular}

Table 4. Comparison within acute biliary pancreatitis between subgroup $\mathrm{A}_{1}$ and $\mathrm{A}_{2}$ with Student's t-test and KolmogorovSmirnov test. 


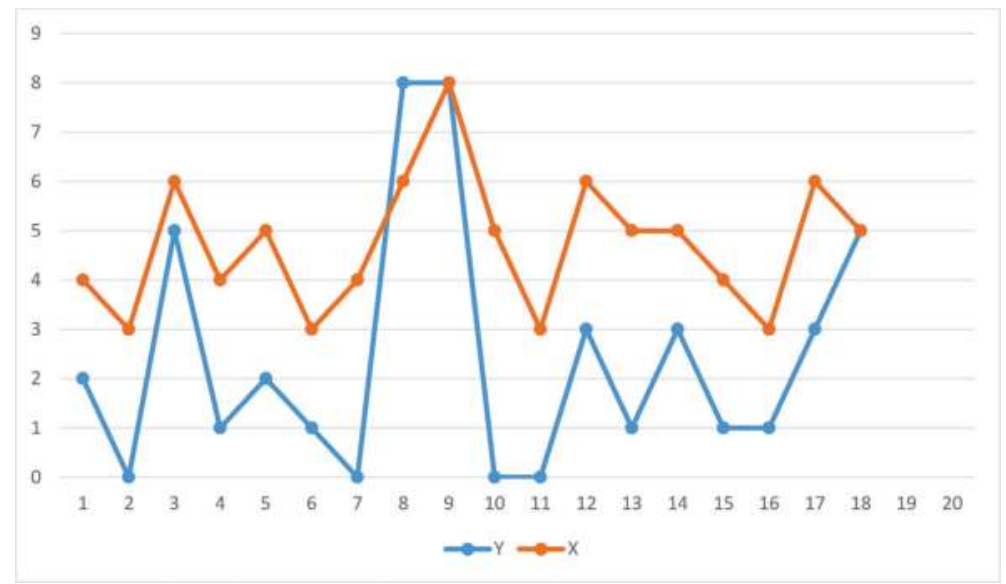

Figure 2. Correspondence between Ranson's score $(\mathrm{X})$ and Balthazar's index $(\mathrm{Y})$ in the A1 subgroup in acute biliary pancreatitis.

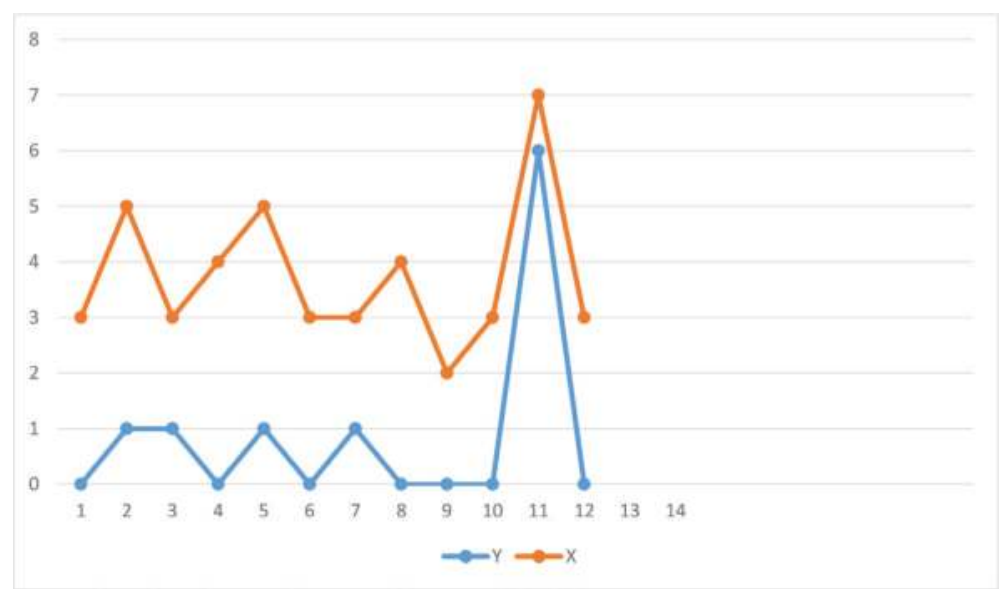

Figure 3. Correspondence between Ranson's score $(\mathrm{X})$ and Balthazar's index $(\mathrm{Y})$ in the A2 subgroup in acute biliary pancreatitis.

pancreatic pattern (subgroup A1), after initial medical treatment, followed by improvement of general conditions and pancreatic involvement, have been treated 7-10 days after onset of disease with cholecystectomy (13 pts.). In five patients with cholestatic index and persistent CBD dilation was planned magnetic resonance cholangiopancreatography (MRCP) and endoscopic retrograde cholangiopancreatography (ERCP)/endoscopic sphincterotomy prior the cholecystectomy, delayed for few days but in the same hospital stay. All cholecystectomies were performed with laparoscopic approach. On the other hand, patients (12) with biliary pattern (subgroup A2) because severe damage of general condition, imminent risk of developing severe sepsis, clinical/instrumental evidence of biliary inflammatory disease 
within acute biliary pancreatitis, underwent emergency surgery (48 hours from onset). The intraoperative findings were acute cholecystitis (6), within two cases choledocolithiasis and cholangitis, in six cases gangrenous cholecystitis. In these patients was present pancreatic edematous impairment. The conversion rate of these procedures was $16.6 \%(2 / 12)$. In both subgroups, postoperative morbidity was Grade I and Grade II according to Clavien-Dindo criteria. In the subgroup A2, mortality rate was $8.3 \%$ (1/12) [23]. We can conclude that it seems possible to identify two types of acute biliary pancreatitis for which the therapeutic approach should be different. The pancreatic pattern characterized by preeminent pancreatic involvement requiring conservative treatment following the severity and evolution of pancreatitis; not delayed cholecystectomy, control, and treatment of papillary obstacle if present, prolonged control of pancreatic and peripancreatic fluid-necrotic collections, and so on. The biliary pattern is characterized by persistent, severe acute biliary tract disease, accompanied by mild or moderate acute biliary pancreatitis. This clinical-pathological condition should undergo urgent surgical intervention to treat the septic-inflammatory disease (acute cholecystitis, cholangitis, etc.).

\section{Pathological features of acute cholecystitis}

Acute cholecystitis can develop some inflammatory complications that give rise to severe pathological conditions, gangrenous cholecystitis, gallbladder empyema, emphysematous cholecystitis, perforation of gallbladder, cholecystoenteric fistula, and gallstone ileus. These complications are life-threatening with risk of severe sepsis and septic shock evolution, peritonitis, and so on; it is mandatory urgent surgical procedure. Gangrenous cholecystitis is a very dangerous complication because of the difficulty of preoperative detection. Gangrene is the development of wall phlogosis, impaired blood supply, wall ischemia, gangrene; the final development of this complication can be the perforation. Gangrene is not frequent complication and perforation can occur in $5-10 \%$ of these patients. Gangrene as complication of acute cholecystitis occurs frequently in patients with compromised clinical conditions: diabetes, trauma, severe burns, prolonged TPN and stay in intensive care unit, cardiac surgery. Frequently perforation is localized in a circumscribed peritonitis, characterized by pericholecystic abscess limited with omentum and surrounding organs. Free perforation causes generalized peritonitis, accompanied by severe impairment of clinical course such as abdominal wall guarding, fever, increase of leukocytosis, start of severe sepsis and septic shock. Gallbladder empyema results as pus collection in the gallbladder because of bacterial overgrow. Obviously, the septic site can initiate severe sepsis and septic shock. The clinical picture is severe with abdominal pain in upper right quadrant, leukocytosis, fever, and tachycardia. Initial medical treatment with broad-spectrum antibiotics must be followed by urgent cholecystectomy. In the emphysematous cholecystitis is added the superinfection of gasforming organisms (Clostridium Welchii, Escherichia coli, Klebsiella, etc.) [24]. This complication is uncommon and usually develops in males, old and diabetics patients. The most frequent evolution of gallbladder wall emphysema (75\% of cases) is gangrene and perforation. Clinical course develops severe sepsis and septic shock. Imagine exams (CT scan) show gas in the gallbladder wall. Emergency surgery should be the correct treatment. 
Cholecystoenteric fistula can be due to dual pathogenesis: long-standing pressure necrosis by large stones and flogistic adhesion of gallbladder wall with adjacent hallow organs, followed by pathological communication. Most frequent communications are with duodenum (70-85\%) and right flexure of the colon (15-20\%) [25]. Cholecystoduodenal fistula allows the passage into the small intestine of gallstones, usually of large size that cause decubital effect. The stone progress in small intestine and in the narrowest part, frequently ileum, stops and determines mechanical obstruction, that is gallstone ileus (about $15 \%$ of patients with cholecystoenteric fistula). Clinical course of gallstone ileus develops as common intestinal obstruction. In the past decades, this clinical condition appeared in the characteristic way: acute cholecystitis treated with medical therapy, in 7-10 days improvement of signs and symptoms (decompression of the gallbladder because the fistula forms), in the following several days appearance of clinical features of intestinal obstruction (gallstone ileus). In the therapeutic program, the first step is the resolution of intestinal obstruction by enterotomy and stone removal. The treatment of cholecystoduodenal fistula should be performed in the same time or delayed for impaired general conditions of patients.

In the uncomplicated cholecystitis, usually there are no increase of serum total and direct bilirubin and alkaline phosphatase (cholestasis indexes). If the signs of cholestasis occur, may be due to choledocolithiasis, cholangitis, or the Mirizzi syndrome. There are two types of Mirizzi syndrome: in type I, a large stone blocked in the cystic duct and in the Hartmann's pouch of gallbladder compresses the common bile duct but without fistula between gallbladder and common hepatic duct. In the type II, due to necrosis of wall of common hepatic duct, there is a fistula with various degrees of defect of hepatic duct wall and presence of stone in hepatic duct. The first type can be treated with "partial" cholecystectomy and repair of bile duct with T-tube. The type II requires more complex procedure with complex dissection and hepaticojejunostomy. On the other hand, there are patients, in course of acute cholecystitis, with mild increase of amylase, AST, ALT, bilirubin caused by papillary passage of sludge, pus, and cholesterol crystals [26]. Moreover in several cases because transient papillary obstruction during transpapillary passage of small stones, can occur elevation of serum transaminase levels (AST, ALT), so called "gallstone hepatitis" [27].

\section{Imaging studies in acute cholecystitis}

\subsection{Plain radiography}

Plain radiography is not very useful to confirm the diagnosis of acute cholecystitis. In few cases, it may detect biliary disease such as biliary stones (only 10-15\% of stones contain calcium enough to be radiopaque), gallbladder wall calcified, pneumobilia; but unfortunately, these findings are not diagnostic for acute cholecystitis. The role of plain radiography remains crucial in any acute abdomen to rule out some pathological condition such as perforated hollow organs (pneumoperitoneum), and intestinal obstruction (air fluid levels). 


\subsection{Ultrasonography}

Transabdominal US should be employed as completion of clinical examination in patients with abdominal pain. It is very important to define accurately the reliable data that the US can provide in different diseases that can cause an acute abdomen. The US can detect gallstones (acoustic shadowing behind to the stones) with sensitivity of $84 \%$ and specificity of $99 \%$ [28-30]. We have to remember some features that US can highlight such as mobile gallstones in the gallbladder, polyps, small stones attached to the wall, very small stones without acoustic shadow, and the fluid absence around the gallstones that make difficult their detection. Finally, there are also some false negative exams with US that range from 5 to $15 \%$ in acute cholecystitis [31, 32]. More crucial for the diagnosis of acute cholecystitis are the gallbladder wall edema or pneumatosis ("double wall sign") and wall thickening; both features of inflammation condition. US can detect bile duct dilation and also the site of obstacle if present. The US can add some information about pericholecystic fluid collection or inflammatory mass in upper right abdominal quadrant but the complete definition of these findings should be obtained by CT scan.

\subsection{Abdominal computed tomography}

CT scan has limited role in the diagnostic confirmation of uncomplicated acute cholecystitis because the same information and sensitivity of US (presence of gallstones, gallbladder wall thickening, dilation of CBD). On the contrary, CT scan is crucial in the diagnostic definition of complications such as pericholecystic fluid, gallbladder empyema, emphysematous gangrene, perforation, limited peritonitis with inflammatory mass, intrahepatic and extrahepatic bile duct dilation, choledocolithiasis, concomitant pancreatitis, hepatic lesions.

\subsection{Magnetic resonance imaging}

Magnetic resonance imaging (MRI) as CT scan is of little help in the diagnosis of simple acute cholecystitis. On the other hand, MRI is very sensitive in detecting the morphology of biliary tract, gallstones, and bile duct stones. Moreover, it is a noninvasive technique in the study of intra- and extrahepatic biliary ducts $[33,34]$. Cholescintigraphy, noninvasive test, allows anatomic and functional evaluation of liver, gallbladder, bile duct. This nuclear medicine exam uses intravenous injection of hepatic 2, 6-dimetyl-imidodiacetic acid (HIDA) that is rapidly excreted in the bile. Cholescintigraphy allows the functional evaluation of hepatic ability to extract the radionuclide, the flow into the biliary ducts and gallbladder and finally the passage into the duodenum within 30-60 min. In the acute cholecystitis, cystic duct obstruction by stones prevents to visualize the gallbladder; also, stones in the common bile duct or papillary obstacle prevent the passage of radionuclide into the duodenum. The sensitivity and specificity of HIDA test in detecting acute cholecystitis reach 90-95\% [35]. In our experience, we do not have used this exam that nowadays is less frequently used.

\section{Treatment}

The first approach in patients with acute cholecystitis includes fluid resuscitation, analgesia, suspension of oral intake, nasogastric tube, broad-spectrum antibiotics. This therapeutic scheme, 
while widely shared, may subject to small variations in timing of each therapeutic measures and in the choice of the antibiotic. Should be discussed the use of nasogastric tube if it can be employed widely at the onset of the disease or selectively in case of nausea, vomiting, abdominal distention. Control of abdominal pain is an essential therapeutic target. For this purpose, nonsteroidal anti-inflammatory drugs are widely used for analgesia. These drugs inhibit the activity of cyclooxygenase 1 and 2 (COX-1 and COX-2) with critical reduction of prostaglandins formation. Prostaglandin E2 plays protective role on epithelial cells of gallbladder by secreting mucin; its reduction decreases this mucin production and consequently the distention of gallbladder wall. The therapy with a single broad-spectrum antibiotic can be correct for mild or moderate acute cholecystitis. In the severe cases should be used more selective antibiotics such as imipenem/ cilastatine, third-generation cephalosporine and metronidazole. Bacteria present in acute cholecystitis are frequently Escherichia coli, Enterococcus, Klebsiella, and so on. In the treatment of acute cholecystitis, cholecystectomy plays the central role as standard management. This statement seems seemingly plain and without controversies. There are in the literature several points of wide discussion in which we will report also our experience. The timing of the intervention is very important topic: the choice between early and delayed cholecystectomy with various operative outcomes. The first item to make is to define "early intervention." Within acute cholecystitis, there are several clinical pathological conditions that are the evolution of the inflammatory/ septic process, from mild to severe, life-threatening forms. The reasonable options, always in urgent approach, can vary from emergency to intervention within 24-48-72 hours (early procedures). Another consideration adds uncertainty in the choice of timing of intervention because the dissection difficulties of inflamed operative site, with the possible increase of intraoperative morbidity that can be very severe in both approaches, laparoscopic and open [36].

In our experience about cholecystectomy morbidity, in the group which includes also the acute cholecystitis, we have compared the outcomes in two following periods: first period 2006-2008 and second period 2009-2011. Total morbidity in the second following period was markedly reduced from 18.5 to $9.96 \%(p=0.009)$. With regard to morbidity by incomplete preoperative evaluation and surgical error, we have defined some criteria to increase the control and prevention: acceptable general anesthesia, clear visibility of surgical site, optimal exposition of the hepatic hilum and its structure, control of possible anatomical variations, finally convertion to open cholecystectomy if necessary [37]. Employing, since 2002, of antegrade dissection in laparoscopic cholecystectomy as standard technique allows reduction in intervention time (mean operative time $40 \mathrm{~min}$ ) and decrease of the conversion rate (from 3.4 to $0.8 \%$ ) in the comparison with common retrograde approach [38]. Minor postoperative morbidities as wound infections can be prevented following correct criteria of medications. In our experience, topical antibiotic application may reduce surgical wound infection in umbilical site after laparoscopic cholecystectomy [39]. Concerning the subhepatic collections, in our opinion, the common use of subhepatic drainage after cholecystectomy for acute cholecystitis enables the correct drainage of serous and/or serohematic secretions usually present in the first days in inflamed surgical site.

There are in the literature several reviews regarding the timing of early or delayed cholecystectomy and the comparison of its operative morbidity. Tokyo guidelines suggest a therapeutic program for acute cholecystitis based on precocious severity assessment as guide for treatment choices. Mild acute cholecystitis should undergo early laparoscopic cholecystectomy, within 72 hours from onset with possible improvement of other medical problems. For moderate 
forms also should be performed early cholecystectomy with laparoscopic or open approach (conversion to open following difficult dissection). Severe acute cholecystitis can show, in addition, damage of general conditions (organ dysfunction) which needs to treat. For these clinical-pathological conditions, urgent surgery is necessary: the type of surgical procedures is connected with pathological findings such as gangrenous or perforated cholecystitis, local or generalized peritonitis, involvement of adjacent organs. The urgent procedures vary from cholecystecyomy to cholecystostomy, percutaneous gallbladder drainage, and so on. The revision of Tokyo guidelines [40] confirms the first choice of laparoscopic early cholecystectomy but without the exact definition of time of precocious intervention. We can underline that in the Tokyo guidelines is reported also the elective cholecystectomy, in all degree of severity, after improvement of the acute inflammatory process [41-43]. Nevertheless, another confirmation of the validity of early cholecystectomy, within 24 hours, regarding minor morbidity and lower cost, has been presented by Gutt CN [44]. More selective criteria have been used in order to bind the study of patients with acute cholecystitis excluding very severe forms (need of intensive care admission, urgent cholecystostomy, etc.) by Canadian Researchers. They employed a population-based analysis (20,000 patients - period 2004-2011) for comparison of operative outcomes of early and delayed cholecystectomy [45].

This study showed, in the comparison of delayed cholecystectomy, that early cholecystectomy in the treatment of acute cholecystitis was associated with a lower risk of major bile duct injury, of operative mortality, of postoperative (30 days) mortality (respectively 1.36 and $0.46 \%$ ) and finally a shorter hospital stay. It is also demonstrated almost same conversion rate between early and delayed laparoscopic cholecystectomy. Obviously early cholecystectomy put in a safe place for risk of recurrent gallstone disease. Similar results have been reported from other studies: early laparoscopic cholecystectomy (performed within 48 hours) is associated to better postoperative outcomes with lower morbidity and hospital stay [46, 47]. A very interesting French study evaluated the choice for optimal timing for early cholecystectomy [48]. Patients with acute cholecystitis from the French National Health Care database have been studied: 42,452 patient -507 hospitals - period 2010-2013. The exam of the literature shows the therapeutic indication of early laparoscopic cholecystectomy as standard procedure for acute cholecystitis. Nevertheless, with exception for urgent surgery indications (sometimes with various procedures) in case of very severe cholecystitis as perforated, gangrenous forms with local or generalized peritonitis, the time of "early surgery" is not well defined. Polo et al. in this study show that the optimal time for laparoscopic cholecystectomy in acute cholecystitis is between the first and third day after hospital admission. In this time interval is recorded lower risk of mortality and lower morbidity: common bile duct injury, reoperation rate, postoperative sepsis, conversion rate, and finally minor length of hospital stay and cost. The definition of best time for surgical procedure always has an element of uncertainty because it is very difficult to report the onset of the symptoms and is instead reported the hospital admission. To assess the significance of this inaccuracy is very difficult. Moreover, this study report also not negligible morbidity and mortality (range from 0.8 to $1.4 \%$ ) for the patients treated within the first 24 hours. This particular result, in our opinion, proves the need, also in the program of early cholecystectomy, of a brief time interval for supportive therapy, and resuscitation to improve the general condition in patients with severe cholecystitis and septic complications. 
It is evident in the recent literature that the first, preferred choice for acute cholecystitis is the laparoscopic approach with conversion rate ranging from 10 to $15 \%$. The first choice of the open approach should be limited to peritonitis, perforated chilecystitis but always as personal choice, that cannot be standardized. In the setting of the therapy of acute cholecystitis, we can propose the presentation of a series of consistent clinical cases, observed and treated in our Institution in the period September 2014-September 2016, to show our treatment program. In the chosen period, we have treated 42 patients with acute cholecystitis. Demographic data are the following: male $45.2 \%$ (19/42), female 54.7\% (23/42), mean age 59.6\% (range: 20-87 years). Furthermore, we have recorded the pathological features of the patients (Table 5).

Therapeutic program in severe cholecystitis with complications, characterized by severe morbidity and mortality, demands ready surgical intervention. In our patients with severe acute cholecystitis (38\%), prompt surgery was performed few hours after hospital admission; two patients needed preoperative intensive care and they were treated within 24 hours. The patients with acute cholecystitis were treated with laparoscopic early cholecystectomy performed within 72 hours (range: few hours-72 hours) based on the needs of preoperative treatments related to comorbidities. All these interventions start with laparoscopic approach and the conversion rate was $21 \%$ (9/42). Postoperative outcomes were characterized by minor morbidity, no mortality and the postoperative hospital stay was in mean 4.2 days (range: 2-14).

Laparoscopic cholecystectomy for acute cholecystitis, because of the inflammation and severe pathological involvement in the operative site, can be in most cases a "difficult cholecystectomy." Our experience and other from the literature have shown that laparoscopic difficult cholecystectomies for acute cholecystitis are safe and effective and are associated with lower incidence of minor and major postoperative complications, moreover with several advantages as less respiratory infections, shorter postoperative course, and shorter hospital stay. Laparoscopic approach decreases some complications of laparotomy as infections, dehiscence, and laparocele [49-51].

There is an impending risk of lesions of common bile duct during cholecystectomy for acute cholecystitis. The adoption of an operating procedure that puts at minor risk biliary duct lesions should be proposed. Gallbladder antegrade dissection is an operative procedure employed also in the past for open cholecystectomy. This well-known type of dissection has been proposed in the laparoscopic approach. Several data from the literature demonstrate the

\begin{tabular}{ll}
\hline Acute cholecystitis & 26 \\
Severe cholecystitis & 16 \\
Hydrope & $(2 / 16)$ \\
Emphysematous & $(3 / 16)$ \\
Gangrene/perforation & $(7 / 16)$ \\
Patients & 42 \\
\hline
\end{tabular}

Table 5. Pathological features in acute cholecystitis (September 2014-September 2016). 
frequent use of this operative procedure in difficult cholecystectomy because of acute flogosis and the decrease in conversion rate to open with antegrade dissection [52, 53].

In our study, we have compared postoperative results of two groups of patients submitted to antegrade dissection and retrograde dissection in laparoscopic approach [54]. This study shows that antegrade dissection in laparoscopy for acute cholecystitis with phlogosis of Calot's triangle is safer procedure in comparison to retrograde approach, seems to reduce the operative time, and should significantly decrease the risk of intraoperative complications such as common bile duct injury and hemorrhages. In our opinion, confirmed by more recent experience, antegrade dissection may be proposed as a standard procedure of cholecystectomy and not only for interventions in the acute cholecystitis [38].

Moreover, there are some specific problems related to urgent cholecystectomy in cirrhotic patients. Cholelithiasis in cirrhotics occurs twice as often in the general population with a reported incidence of $9-13 \%$ versus 5\% in non-cirrhotic patients [55]. Major incidence of cholelithiasis is due to several factors with various pathogenesis: hypersplenism, increased level of estrogen, increased intravascular hemolysis, reduction in gallbladder emptying, and motility. In our experience, published some years ago [56], this epidemiologic characteristic was confirmed. In this study, we have evaluated 65 cirrhotic patients with symptomatic gallstone disease treated with laparoscopic cholecystectomy in the decade 2002-2012. This group of patients has been compared with 81 non-cirrhotic patients with symptomatic gallstone disease, no significant morbidity and no significant differences in demographic data, and underwent laparoscopic cholecystectomy in the period October 2011-May 2012. Within the group of non-cirrhotic patients, the incidence of acute cholecystitis was 13.5\% (11/81) and there are not further complications; on the contrary, in the cirrhotic group, the incidence of acute cholecystitis reached $27.6 \%(18 / 65)$ with several complications such as 1 cholangitis, 2 gallbladder hydrops, 2 gallbladder empyema, 3 gangrenous cholecystitis. The cohort of cirrhotics evaluated by Child-Pough classification shows 43 patients in A score (66.2\%), 19 in B score $(29.2 \%)$, and three patients in C score, medically treated preoperatively and reclassified in B8 score. Cirrhotic patients have undergone cholecystectomy: six with open approach as first choice, 59 with laparoscopic procedure with conversion rate $20.3 \%$. The comparison of the results between the two cohorts of patients has been evaluated (Table 6).

In this experience, laparoscopic cholecystectomy morbidity in cirrhotic patients is slightly increased compared to non-cirrhotics. Moreover, postoperative morbidity in cirrhotic patients is minor on the whole with laparoscopic approach than open procedure. Cholecystectomy in cirrhotic patients is associated with non-negligible rate of morbidity and mortality. The more frequent complications are blood loss, postoperative liver failure, and sepsis [57, 58]. Postoperative liver failure is due to the anesthetic agent's action, which decrease hepatic arterial blood flow (the ability of cirrhotic patients to compensate for this ischemia is impaired) [59]. Diminished Kupffer cell function leads to reduced clearance of the enteric organisms, endotoxinemia, and risk of infection in cirrhotic patients. The increased risk of bleeding is related to reduced prothrombin time, thrombocytopenia, and portal ipertension. Finally, patients can have a gallbladder with a significant intrahepatic component due to atrophy of the right hepatic lobe and a hypertrophic left lobe with more difficulties for intervention [55, 60-62]. 


\begin{tabular}{lllll}
\hline & VLC (47) & Open (6) & Converted (12) & 85 \\
\hline Operative time (minutes) & 88.9 & 141 & 149 & 3.2 \\
Hospitalization (days) & 4.8 & 9.1 & 8.1 & $3(3.7 \%)$ \\
Conversion rate & $20.34 \%$ & - & - & - \\
Mortality & - & - & - & - \\
Blood transfusion in peri-operative time & $2(4.2 \%)$ & $1(16.6 \%)$ & $2(16.6 \%)$ & - \\
Blood products transfusion in peri- & $7(14.9 \%)$ & $1(16.6 \%)$ & $4(33.3 \%)$ & - \\
operative time & & & - & - \\
Hemoperitoneum & $1(2.12 \%)$ & - & - & - \\
Reintervention & $1(2.12 \%)$ & - & $2(16.6 \%)$ & - \\
Pleural effusion & - & - & $1(8.33 \%)$ & - \\
Pulmonary condensation & - & - & $1(8.33 \%)$ & - \\
Trombocytopenia & - & - & - & $2(2.46 \%)$ \\
Atrial fibrillation & - & - & \\
Incisional hernia on umbilical port site & $4(8.51 \%)$ & & - \\
\hline
\end{tabular}

Table 6. Peri- and postoperative morbidity outcomes in cirrhotics (65) and in control group (81).

The last evolution in the surgical treatment of acute cholecystitis is the robotic approach. On the whole, the main advantages of robotic surgery can be realized in some phases of complex laparoscopic procedures requiring high dexterity and best visualization. In this perspective, robotic approach for cholelithiasis and later for acute cholecystitis should be the start of valuable learning curve for robotic advanced skills in general surgery. Our experience in the field of gallbladder lithiasis confirms the safe feasibility of robotic approach that requires the use of standardized procedures. The obvious purpose of this approach, however, in the cholecystectomy, is the improvement of the technical skills in advanced and more complex robotic assisted surgical procedures [63]. The comparison of the results of laparoscopic versus robotic cholecystectomy proves the complete equivalence between both the procedures regarding of safety and feasibility in all types of gallbladder's pathology. In particular, acute cholecystitis can be treated with robotic-assisted approach showing postoperative overlapping outcomes with symptomatic gallstones disease [64]. On the contrary, the data from a study based on the literature search with randomized controlled trials and population-based analyses shows that the advantages of current use of robotic surgery in cholecystectomy are not provable [65].

\section{Conclusions}

Acute cholecystitis encompasses clinical forms with various degree of severity and several cases $(8-10 \%)$ present pathological findings that can make the operative site a surgical challenge, very difficult to treat. Indeed the laparoscopic approach, worldwide more common 
choice in the treatment of acute cholecystitis, presents significant conversion rate to open procedure (10-15\%). Furthermore, besides more common clinical, laboratory, and instrumental features of acute cholecystitis, there are some diagnostic pitfalls, such as the biliary pattern that should be distinguished from the pancreatic pattern in the field of acute biliary pancreatitis. The treatment is focused on early laparoscopic cholecystectomy well defined usually within 24-72 hours. Nevertheless, severe, complicated acute cholecystitis can require urgent surgical intervention. Finally should be evaluated some particular components of a complex clinical problem such as laparoscopic antegrade dissection in acute cholecystitis to allow minor risk of biliary duct lesions, the control of the specific problems related to urgent cholecystectomy in cirrhotic patients, and finally the possible future increased use of robotic approach in the treatment of acute cholecystitis.

\section{Author details}

Pasquale Cianci, Nicola Tartaglia, Alberto Fersini, Sabino Capuzzolo, Libero Luca Giambavicchio, Antonio Ambrosi and Vincenzo Neri*

*Address all correspondence to: vincenzo.neri@unifg.it

Department of Medical and Surgical Sciences, University of Foggia, Foggia, Italy

\section{References}

[1] Shaffer EA. Epidemiology and risk factors for gallstone disease: Has the paradigm changed in the 21st century?. Curr Gastroenterol Rep. 2005;7(2):132-40.

[2] Tazuma S. Gallstone disease: Epidemiology, pathogenesis, and classification of biliary stones (common bile duct and intrahepatic). Best Pract Res Clin Gastroenterol. 2006;20(6):1075-83.

[3] Everhart JE, Ruhl CE. Burden of digestive diseases in the United States part I: Overall and upper gastrointestinal diseases. Gastroenterology. 2009;136(2):376-86. doi:10.1053/j. gastro.2008.12.015.

[4] Shaheen NJ, Hansen RA, Morgan DR, Gangarosa LM, Ringel Y, Thiny MT, Russo MW, Sandler RS. The burden of gastrointestinal and liver diseases, 2006. Am J Gastroenterol. 2006;101(9):2128-38.

[5] Lindkvist B, Appelros S, Manjer J, Borgström A. Trends in incidence of acute pancreatitis in a Swedish population: Is there really an increase?. Clin Gastroenterol Hepatol. 2004;2(9):831-7.

[6] Zha Y, Chen XR, Luo D, Jin Y. The prevention of major bile duct injures in laparoscopic cholecystectomy: The experience with 13,000 patients in a single center. Surg Laparosc Endosc Percutan Tech. 2010;20(6):378-83. doi:10.1097/SLE.0b013e3182008efb. 
[7] Admirand WH, Small DM. The physicochemical basis of cholesterol gallstone formation in man. J Clin Invest. 1968;47(5):1043-52.

[8] He J, Nishida S, Xu M, Makishima M, Xie W. PXR prevents cholesterol gallstone disease by regulating biosynthesis and transport of bile salts. Gastroenterology. 2011;140(7):2095-106. doi:10.1053/j.gastro.2011.02.055.

[9] Glasgow RE, Cho M, Hutter MM, Mulvihill SJ. The spectrum and cost of complicated gallstone disease in California. Arch Surg. 2000;135(9):1021-5; discussion 1025-7.

[10] Csendes A, Burdiles P, Maluenda F, Diaz JC, Csendes P, Mitru N. Simultaneous bacteriologic assessment of bile from gallbladder and common bile duct in control subjects and patients with gallstones and common duct stones. Arch Surg. 1996;131(4):389-94.

[11] Roslyn JJ, DenBesten L, Thompson JE Jr, Silverman BF. Roles of lithogenic bile and cystic duct occlusion in the pathogenesis of acute cholecystitis. Am J Surg. 1980;140(1):126-30.

[12] Kaminski DL. Arachidonic acid metabolites in hepatobiliary physiology and disease. Gastroenterology. 1989;97(3):781-92.

[13] Jivegård L, Thornell E, Svanvik J. Pathophysiology of acute obstructive cholecystitis: Implications for non-operative management. Br J Surg. 1987;74(12):1084-6.

[14] Barie PS, Fischer E. Acute acalculous cholecystitis. J Am Coll Surg. 1995;180(2):232-44.

[15] Osborne DA, Alexander G, Boe B, Zervos EE. Laparoscopic cholecystectomy: Past, present, and future. Surg Technol Int. 2006;15:81-5.

[16] Hansel SL, DiBaise JK. Functional gallbladder disorder: Gallbladder dyskinesia. Gastroenterol Clin North Am. 2010;39(2):369-79, x. doi:10.1016/j.gtc.2010.02.002.

[17] Singer AJ, McCracken G, Henry MC, Thode HC Jr, Cabahug CJ. Correlation among clinical, laboratory, and hepatobiliary scanning findings in patients with suspected acute cholecystitis. Ann Emerg Med. 1996;28(3):267-72.

[18] Isogai M, Hachisuksa K, Yamaguchi A, Nakano S. Clinical diversity in biliary pancreatitis: Classification of two types. HPB Surg. 1993;6(4):263-75; discussion 275-6.

[19] Ranson JH, Rifkind KM, Roses DF, Fink SD, Eng K, Spencer FC. Prognostic signs and the role of operative management in acute pancreatitis. Surg Gynecol Obstet. 1974;139(1):69-81.

[20] Balthazar EJ. Acute pancreatitis: Assessment of severity with clinical and CT evaluation. Radiology. 2002;223(3):603-13.

[21] Ranson JH, Pasternack BS. Statistical methods for quantifying the severity of clinical acute pancreatitis. J Surg Res. 1977;22(2):79-91.

[22] Blamey SL, Imrie CW, O'Neill J, Gilmour WH, Carter DC. Prognostic factors in acute pancreatitis. Gut. 1984;25(12):1340-6. 
[23] Clavien PA, Barkun J, de Oliveira ML, Vauthey JN, Dindo D, Schulick RD, de Santibañes E, Pekolj J, Slankamenac K, Bassi C, Graf R, Vonlanthen R, Padbury R, Cameron JL, Makuuchi M. The Clavien-Dindo classification of surgical complications: 5 year experience. Ann Surg. 2009;250(2):187-96. doi:10.1097/SLA.0b013e3181b13ca2.

[24] Lorenz RW, Steffen HM. Emphysematous cholecystitis: Diagnostic problems and differential diagnosis of gallbladder gas accumulations. Hepatogastroenterology. 1990; 37(Suppl 2):103-6.

[25] Roslyn JJ, Thompson JE Jr, Darvin H, DenBesten L. Risk factors for gallbladder perforation. Am J Gastroenterol. 1987;82(7):636-40.

[26] Kurzweil SM, Shapiro MJ, Andrus CH, Wittgen CM, Herrmann VM, Kaminski DL. Hyperbilirubinemia without common bile duct abnormalities and hyperamylasemia without pancreatitis in patients with gallbladder disease. Arch Surg. 1994;129(8):829-33.

[27] Isogai M, Hachisuka K, Yamaguchi A, Nakano S. Etiology and pathogenesis of marked elevation of serum transaminase in patients with acute gallstone disease. HPB Surg. 1991;4(2):95-105; discussion 106-7.

[28] Rosen CL, Brown DF, Chang Y, Moore C, Averill NJ, Arkoff LJ, McCabe CJ, Wolfe RE. Ultrasonography by emergency physicians in patients with suspected cholecystitis. Am J Emerg Med. 2001;19(1):32-6.

[29] Shea JA, Berlin JA, Escarce JJ, Clarke JR, Kinosian BP, Cabana MD, Tsai WW, Horangic N, Malet PF, Schwartz JS, et al. Revised estimates of diagnostic test sensitivity and specificity in suspected biliary tract disease. Arch Intern Med. 1994;154(22):2573-81.

[30] Rubens DJ.Hepatobiliary imaging and its pitfalls. Radiol Clin North Am. 2004;42(2):257-78.

[31] Shah K, Wolfe RE. Hepatobiliary ultrasound. Emerg Med Clin North Am. 2004;22(3): 661-73, viii.

[32] Pereira J, Afonso AC, Constantino J, Matos A, Henriques C, Zago M, Pinheiro L. Accuracy of ultrasound in the diagnosis of acute cholecystitis with coexistent acute pancreatitis. Eur J Trauma Emerg Surg. 2015;43. [Epub].

[33] Kim YK, Kwak HS, Kim CS, Han YM, Jeong TO, Kim IH, Yu HC. CT findings of mild forms or early manifestations of acute cholecystitis. Clin Imag. 2009;33(4):274-80. doi:10.1016/j.clinimag.2008.11.004.

[34] Wang A, Shanbhogue AK, Dunst D, Hajdu CH, Rosenkrantz AB. Utility of diffusionweighted MRI for differentiating acute from chronic cholecystitis. J Magn Reson Imag. 2016;44(1):89-97. doi:10.1002/jmri.25128. [Epub 22 Dec 2015].

[35] Kim EE, Moon TY, Delpassand ES, Podoloff DA, Haynie TP. Nuclear hepatobiliary imaging. Radiol Clin North Am. 1993;31(4):923-33.

[36] Strasberg SM. Clinical practice. Acute calculous cholecystitis. N Engl J Med. 2008;358: 2804-11. doi:10.1056/NEJMcp0800929. 
[37] Neri V, Lapolla F, Forlano I, Di Lascia A, Fersini A, Tartaglia N. Cholecystectomy morbidity in the laparoscopic era. Wyno J Med Sci. 2013;2(2):19-25.

[38] Tartaglia N, Cianci P, Di Lascia A, Fersini A, Ambrosi A, Neri V. Laparoscopic antegrade cholecystectomy: A standard procedure? De Gruyter Open Med. 2016;11:429-32.

[39] Neri V, Fersini A, Ambrosi A, Tartaglia N, Valentino TP. Umbilical port-site complications in laparoscopic cholecystectomy: Role of topical antibiotic therapy. JSLS. 2008;12(2):126-32.

[40] Miura F, Takada T, Strasberg SM, Solomkin JS, Pitt HA, Gouma DJ, Garden OJ, Büchler MW, Yoshida M, Mayumi T, Okamoto K, Gomi H, Kusachi S, Kiriyama S, Yokoe M, Kimura Y, Higuchi R, Yamashita Y, Windsor JA, Tsuyuguchi T, Gabata T, Itoi T, Hata J, Liau KH; Tokyo Guidelines Revision Comittee. TG13 flowchart for the management of acute cholangitis and cholecystitis. J Hepatobiliary Pancreat Sci. 2013;20(1):47-54. doi:10.1007/ s00534-012-0563-1.

[41] Miura F, Takada T, Kawarada Y, Nimura Y, Wada K, Hirota M, Nagino M, Tsuyuguchi T, Mayumi T, Yoshida M, Strasberg SM, Pitt HA, Belghiti J, de Santibanes E, Gadacz TR, Gouma DJ, Fan ST, Chen MF, Padbury RT, Bornman PC, Kim SW, Liau KH, Belli G, Dervenis C. Flowcharts for the diagnosis and treatment of acute cholangitis and cholecystitis: Tokyo guidelines. J Hepatobiliary Pancreat Surg. 2007;14(1):27-34.

[42] Sugiyama M, Tokuhara M, Atomi Y. Is percutaneous cholecystostomy the optimal treatment for acute cholecystitis in the very elderly? World J Surg. 1998;22(5):459-63.

[43] Gutt CN, Encke J, Köninger J, Harnoss JC, Weigand K, Kipfmüller K, Schunter O, Götze T, Golling MT, Menges M, Klar E, Feilhauer K, Zoller WG, Ridwelski K, Ackmann S, Baron A, Schön MR, Seitz HK, Daniel D, Stremmel W, Büchler MW. Acute cholecystitis: Early versus delayed cholecystectomy, a multicenter randomized trial (ACDC study, NCT00447304). Ann Surg. 2013;258(3):385-93. doi:10.1097/SLA.0b013e3182a1599b.

[44] Chopra S, Dodd GD 3rd, Mumbower AL, Chintapalli KN, Schwesinger WH, Sirinek KR, Dorman JP, Rhim H. Treatment of acute cholecystitis in non-critically ill patients at high surgical risk: Comparison of clinical outcomes after gallbladder aspiration and after percutaneous cholecystostomy. AJR Am J Roentgenol. 2001;176(4):1025-31.

[45] de Mestral C, Rotstein OD, Laupacis A, Hoch JS, Zagorski B, Alali AS, Nathens AB. Comparative operative outcomes of early and delayed cholecystectomy for acute cholecystitis: A population-based propensity score analysis. Ann Surg. 2014;259(1):10-5. doi:10.1097/SLA.0b013e3182a5cf36.

[46] Banz V, Gsponer T, Candinas D, Güller U. Population-based analysis of 4113 patients with acute cholecystitis: Defining the optimal time-point for laparoscopic cholecystectomy. Ann Surg. 2011;254(6):964-70. doi:10.1097/SLA.0b013e318228d31c.

[47] Brooks KR, Scarborough JE, Vaslef SN, Shapiro ML. No need to wait: An analysis of the timing of cholecystectomy during admission for acute cholecystitis using the American 
College of Surgeons National Surgical Quality Improvement Program database. J Trauma Acute Care Surg. 2013;74(1):167-73; 173-4. doi:10.1097/TA.0b013e3182788b71.

[48] Polo M, Duclos A, Polazzi S, Payet C, Lifante JC, Cotte E, Barth X, Glehen O, Passot G. Acute cholecystitis-optimal timing for early cholecystectomy: A French nationwide study. J Gastrointest Surg. 2015;19(11):2003-10. doi:10.1007/s11605-015-2909-x.

[49] Neri V, Ambrosi A, Di Lauro G, Fersini A, Valentino TP. Difficult cholecystectomies: Validity of the laparoscopic approach. JSLS. 2003;7(4):329-33.

[50] Amaral PC, Azaro Filho EM, Galvão-Neto MP, Fortes MF, Souza EL, Alcântra RS, Ettinger JE, Regis AB, Sousa MM, do Carmo VM, Santana PA Jr, Fahel E. Acute cholecystitis: Video-laparoscopic versus traditional treatment. JSLS. 2001;5(2):159-65.

[51] Pisanu A, Floris G, Ambu R, Uccheddu A. Early surgical treatment of acute cholecystitis. A retrospective comparative study of the laparoscopic and open approaches. Chir Ital. 2001;53(2):159-65.

[52] Gupta A, Agarwal PN, Kant R, Malik V. Evaluation of fundus-first laparoscopic cholecystectomy. JSLS. 2004;8(3):255-8.

[53] Rosenberg J, Leinskold T. Dome down laparosonic cholecystectomy. Scand J Surg. 2004;93(1):48-51.

[54] Neri V, Ambrosi A, Fersini A, Tartaglia N, Valentino TP. Antegrade dissection in laparoscopic cholecystectomy. JSLS. 2007;11(2):225-8.

[55] Machado NO. Laparoscopic cholecystectomy in cirrhotics. JSLS. 2012;16(3):392-400. doi: $10.4293 / 108680812 X 13462882736493$.

[56] Neri V, Forlano I, Lapolla F, Fersini A, Tartaglia N. Laparoscopic cholecystectomy in cirrhotic patients. Sch J App Med Sci. 2013;1(5):637-44.

[57] Fernandes NF, Schwesinger WH, Hilsenbeck SG, Gross GW, Bay MK, Sirinek KR, Schenker S. Laparoscopic cholecystectomy and cirrhosis: A case-control study of outcomes. Liver Transpl. 2000;6(3):340-4.

[58] Bernardo WM, Aires FT. Is laparoscopic cholecystectomy safe in patients with liver cirrhosis? Rev Assoc Med Bras (1992). 2011;57(4):360-1.

[59] Gugenheim J, Casaccia M Jr, Mazza D, Toouli J, Laura V, Fabiani P, Mouiel J. Laparoscopic cholecystectomy in cirrhotic patient. HPB Surg. 1996;10(2):79-82.

[60] Puggioni A, Wong LL. A metaanalysis of laparoscopic cholecystectomy in patients with cirrhosis. J Am Coll Surg. 2003;197(6):921-6.

[61] Currò G, Iapichino G, Melita G, Lorenzini C, Cucinotta E. Laparoscopic cholecystectomy in Child-Pugh class C cirrhotic patients. JSLS. 2005;9(3):311-5. 
[62] Pavlidis TE, Symeonidis NG, Psarras K, Skouras C, Kontoulis TM, Ballas K, Rafailidis SF, Marakis GN, Sakantamis AK. Laparoscopic cholecystectomy in patients with cirrhosis of the liver and symptomatic cholelithiasis. JSLS. 2009;13(3):342-5.

[63] Tartaglia N, Di Lascia A, Vovola F, Cianci P, Fersini A, Ambrosi A, Neri V. Robotic cholecystectomy: Preliminary experience and future perstectives. JSM Gen Surg Cases Images. 2016;1(1):1003.

[64] Ayloo S, Roh Y, Choudhury N. Laparoscopic versus robotassisted cholecystectomy: A retrospective cohort study. Int J Surg. 2014;12:1077-81.

[65] Koti RS, Davidson CJ, Davidson BR. Surgical management of acute cholecystitis. Langenbecks Arch Surg. 2015;400(4):403-19. doi:10.1007/s00423-015-1306-y. [Epub 14 May 2015]. 
